\title{
Iliotibial Band Friction Syndrome: A Systematic Review and Meta-analysis to evaluate lower-limb biomechanics and conservative treatment
}

\author{
V. Balachandar ${ }^{1}$, M. Hampton ${ }^{1}$, O. Riaz'2, S. Woods² \\ 1 Northern General Hospital, Sheffield Teaching Hosptials, Sheffield, UK. \\ 2 Leeds General Infirmary, Leeds Teaching Hospitals, Leeds, UK
}

\section{CORRESPONDING AUTHOR:}

Vivek Balachandar

Northern General Hospital,

Sheffield Teaching Hoispitals

Sheffield, UK

E-mail: vbsheffield1@gmail.com

Phone: 07745678360

DOI:

10.32098/mltj.02.2019.05

LEVEL OF EVIDENCE:

$1 a$

\begin{abstract}
SUMMARY
Objective. Iliotibial band friction syndrome (ITBFS) is the second most common cause of knee pain in runners, and the most common cause of lateral knee pain. This systematic review and meta-analysis evaluates 1) lower-limb biomechanics and 2) conservative interventions in ITBFS. Methods. The MEDLINE, CINAHL, SPORTSDiscus, Web of Science, and Google Scholar databases were searched in April 2018 for studies investigating lower limb biomechanics and conservative treatment in iliotibial band friction syndrome. Results. Eighteen studies were identified. Moderate evidence from prospective studies indicate 1) greater hip adduction and knee internal rotation (IR) at footstrike and through stance, and 2) greater rearfoot eversion at foot strike represent possible mechanisms for ITBFS. Moderate evidence from case-control studies indicate ITBFS participants exhibit 1) reduced hip adduction, 2) greater knee and hip IR at footstrike and through stance, and 3) reduced rearfoot eversion at foot strike. Moderate evidence indicates that a six-week rehabilitation programme involving NSAID prescription, ITB stretching, and hip abductor strengthening reduces knee pain during physical activity, and prevents ITBFS recurrence in the medium term ( $\leq 6$ months). Conclusion. Greater hip adduction, knee IR, and rearfoot eversion represent possible risk factors for ITBFS. Greater hip adduction and rearfoot eversion appear to be risk factors which are reduced by ITBFS patients, to lower pain associated with ITB strain, ITB friction and compression. Addressing the absence of research evaluating lower limb electromyography is needed to understand the influence of muscle function on ITBFS. A six-week rehabilitation programme involving NSAID prescription, ITB stretching, and hip abductor strengthening effectively reduces pain and prevents recurrence for up to six months.
\end{abstract}

KEY WORDS

Systematic review; iliotibial band; biomechanics

\section{INTRODUCTION}

Iliotibial band friction syndrome (ITBFS) is the second most common cause of knee pain in runners, and accounts for up to $12 \%$ of all running injuries. $(1,2)$ Simple activities of daily living such as squatting, walking down stairs, and driving commonly exacerbate ITBFS, thereby reducing occupational and physical capacity. (2)
ITBFS is a syndrome associated with overuse, and is therefore most common in active individuals. ${ }^{(3)}$ The condition is characterised by lateral knee pain, and typically occurs between 20-30 knee flexion. (2) Repetitive irritation of the iliotibial band (ITB) can lead to chronic inflammation and swelling over the lateral femoral epicondyle (LFE).(2) The ITB is a thick sheet of connective tissue originating at the 
anterior superior iliac spine (ASIS) from fascial connections of the gluteus maximus (GM) and the tensor fascial lata (TFL).(4) The band passes distally down the lateral aspect of the pelvis and femur, inserting at the LFE, lateral patellar, and most distally at the infracondylar tubercle of the tibia (Gerty's tubercle) and to the head of the fibula.(4) The attachments of the ITB mean that it passively resists hip adduction and anterior translation and internal rotation of the tibia.(4)

The aetiology of ITBFS is multifactorial, with both extrinsic and intrinsic factors thought to contribute. (1) Considering the anatomical and biomechanical associations with ITBFS, a number of studies have investigated lower-limb kinematics (hip, knee, ankle, and foot), and kinetics (ground reaction forces, joint moments, and tissue strain) in ITBFS. Traditional theories proposed that greater ITB friction over the LFE during running may result in chronic inflammation and the formation of an underlying bursa between the LFE and ITB with associated pain. However, recent studies suggest that pain may be the result of repeated ITB compression against a local adipose fat pad rather than friction. $(2,4) \mathrm{A}$ systematic review by Louw (5) proposed that a combination of greater hip adduction and internal rotation of the femur may increase ITB strain during running. Given that abnormal lower-limb biomechanics during running may increase ITB strain, this may result in greater ITB friction and/or compression and associated pain. Considering the biomechanical associations with ITBFS, conservative interventions such as stretching and strengthening exercises form core components in rehabilitation programmes. Ellis et al (6) published a systematic review on conservative treatment for ITBFS until September 2004 and concluded there was insufficient evidence to suggest that conservative treatments offered any significant benefit in the management of ITBFS. In 2018, previous systematic reviews by Louw, (5) Worp, (7) Ellis (6) have at least four limitations. First, all three studies are now significantly dated, and do not benefit from five important recent trials concerning biomechanical analysis and conservative interventions. (8-12) Second, in Ellis's systematic review, no studies investigating treatments targeted at altering lower-limb biomechanics were included.(6) Third, no reviews have pooled study data to calculate effect-sizes for outcome variables. Fourth, Worp's review of biomechanics in ITBFS upto 2012 used very stringent inclusion criteria and thus included just four trials. (7) Although stringency is laudable, the review concluded that there was insufficient evidence regarding lower-limb biomechanical associations with ITBFS. Our systematic review and meta-analysis was designed to loosen Worp's criteria slightly while still using clear principles taking into account risk of bias in lower-quality studies when determining levels of evidence. Our aims included 1) evaluation of lower-limb biomechanics and 2) efficacy of conservative treatment in individuals with ITBFS.

\section{METHODS}

The PRISMA statement was consulted and completed prior to the start of this review and the checklist completed. (14)

\section{Search Strategy}

MEDLINE, CINAHL, SPORTSDiscus (SD), Web of Science (WoS), and Google Scholar databases were searched from inception until April 2018. Reference lists of included studies were screened, key words searched, and search results shown in appendix table 1 .

\section{Inclusion and Exclusion Criteria}

Prospective and case-control studies evaluating lower-limb kinematics and kinetics during functional activity in adults (18-50) who developed ITBFS or adults with ITBFS were included. Studies evaluating the efficacy of conservative interventions targeted at improving lower-limb biomechanics in adults with ITBFS were also included. Other terms associated with ITBFS (e.g. iliotibial band syndrome) were also included. Iliotibial band friction syndrome was considered too narrow a search field, therefore only 'iliotibial' was used. Unpublished studies, case-reports, non-peer reviewed publications, studies not involving humans, reviews, letters, and opinion articles were excluded. Studies including participants with other knee conditions such as meniscal injury, and studies only investigating the efficacy of interventions not aimed at improving lower-limb biomechanics such as NSAIDs and deep-tissue friction massage were excluded.

\section{Review Process}

All retrieved studies were downloaded to Endnote version X4. Results were cross-referenced and duplicated studies were deleted. Relevant titles were highlighted, with abstracts and full texts reviewed independently for inclusion (appendix figure 1)

\section{Quality Assessment of Studies}

All potential studies were assessed for inclusion by two independent reviewers. Included studies were then assessed by the same independent reviewers for quality using the PEDRO scale (table 1), (15) and the modified Down and Black scale (table 2). (16) Any discrepancy between the 
reviewers on assessment were resolved through a consensus meeting, with a third independent reviewer available but not required. Following application of the PEDro scale, studies with a score of $\geq 8 / 10$ were considered of very high quality, 5-7/10 high, and $\leq 4 / 10$ low. (16) With the modified Down's and Black scale, studies scoring $\geq 11 / 14$ were considered of very high quality, $7-10 / 14$ high, and $\leq 6 / 14$ low.(15)

To assist with interpretation of findings, information including study design, population (number of participants, mean age), intervention, control intervention/comparisons, and results were extracted from each included study (table 3). Means and standard deviations for all baseline and followup data were extracted to calculate effect sizes (Cohen's d) using Cochrane Review Manager (Version 5) where effect sizes were small $(<0.6)$, medium (0.6-1.19), and large $(>1.2)$. Where means and standard deviations were not available in the publication, corresponding authors were contacted via email to seek additional data. For ease of visual interpretation, effect size calculations including 95\% confidence intervals were presented in forest plots produced by the same Review Manager software. Where study homogeneity (i.e. similar outcome measures) existed, data pooling was performed. The strength of evidence supporting each outcome was then determined by the number and quality of studies supporting that finding using pre-determined criteria proposed by van Tulder et al:(16) 'Strong evidence' is based on results derived from three or more studies, including a minimum of two high-quality studies which are statistically homogonous $(p>0.05)$ with or without statistically significant pooled results. 'Moderate evidence' is based on results derived from multiple studies, including at least one high-quality study which are statistically heterogeneous $(\mathrm{p}<0.05)$, or from low quality studies which are statistically homogenous $(p>0.05)$. 'Limited evidence' includes results from multiple low-quality studies which are statistically heterogeneous $(\mathrm{p}<0.05)$ or from one high-quality study. 'Very limited evidence' is based on results from one low-quality study. 'Conflicting evidence' includes insignificant pooled results derived from multiple studies regardless of quality which are statistically heterogeneous $(\mathrm{p}<0.05)$.

\section{RESULTS}

\section{Search Results}

The initial search produced upto 903 citations. Following application of the selection criteria to titles and abstracts this was reduced to 26 , and after viewing full texts the final number was 18 . The main reason for exclusion was due to studies investigating non-conservative interventions or interventions not aimed at improving lower-limb biomechanics.
The majority of studies were consistent in their inclusion of ITBFS participants, however, only four studies performed and reported a positive Ober's test. $(17,18,21,23)$

Results of the PEDro and modified Down's and Black scales are shown in appendix table 1 and 2. Appendix table 3 summarises the included studies and methodological criteria. Eleven studies in this review investigated lower-limb kinematics, (8-12,17-20,22,27) eleven lower-limb kinetics, $(9,11,17-24,27)$ and four conservative treatments. $(23-26)$ Eleven studies presented adequate data to complete effect size calculations $(95 \% \mathrm{CI}),(10,11,17-21,23,24,26,27)$ and none provided further data. The studies were separated into three main outcome measure categories for further review: i) prospective studies; lower-limb kinematics, kinetics, ITB strain, ii) case-control studies; lower-limb kinematics, kinetics, and iii) conservative interventions; longer-term, mid-term, short-term, and immediate improvements.

\section{Prospective studies}

Lower-limb kinematics in individuals who develop ITBFS Limited evidence from one high quality study indicates significantly greater hip adduction at landing and during running with medium effect in individuals who developed ITBFS compared to controls $(\mathrm{ES}=0.85)$. (20)

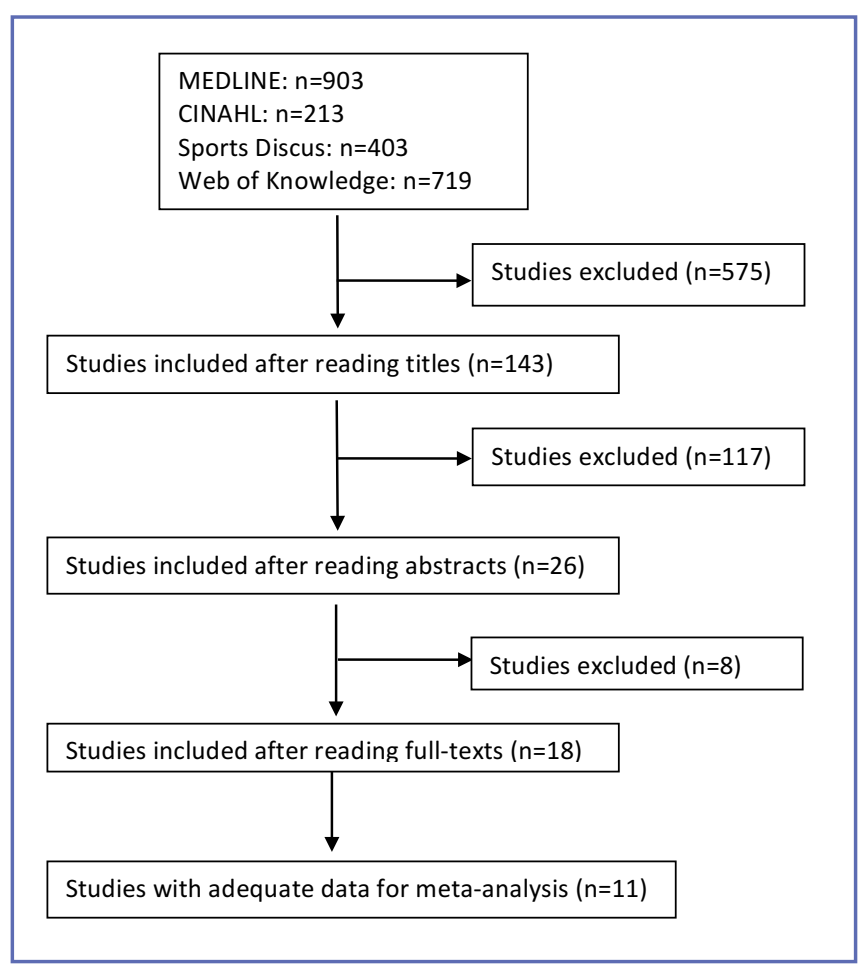

Figure 1: Search strategy with outcomes at each stage 
Table 1: PEDRO scale

\begin{tabular}{|c|c|c|c|c|c|c|c|c|c|c|c|c|}
\hline Paper & 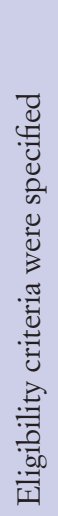 & 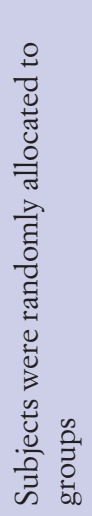 & 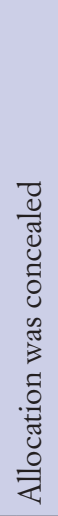 & 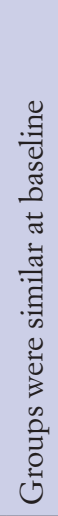 & 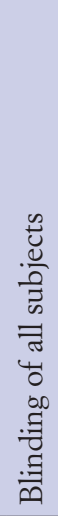 & 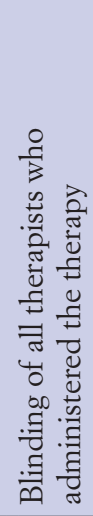 & 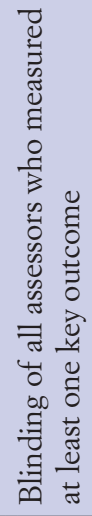 & 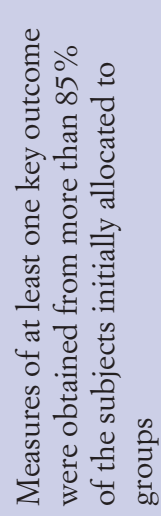 & 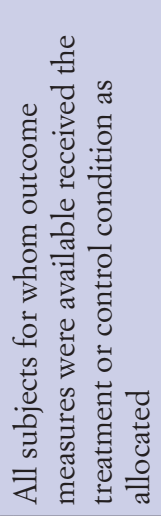 & 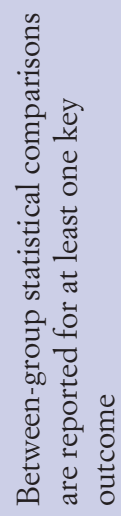 & 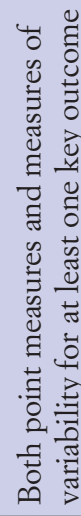 & $\underset{\mathrm{O}}{\stackrel{\xi}{\xi}}$ \\
\hline Fredericson & 1 & 1 & 0 & 1 & 0 & 0 & 0 & 1 & 1 & 1 & 1 & 7 \\
\hline Beers & 1 & 0 & 0 & 0 & 0 & 0 & 0 & 1 & 1 & 1 & 1 & 6 \\
\hline Ekman & 1 & 0 & 0 & 0 & 0 & 0 & 0 & 1 & 1 & 1 & 1 & 5 \\
\hline Orchard & 1 & 0 & 0 & 0 & 0 & 0 & 0 & 1 & 1 & 1 & 1 & 5 \\
\hline
\end{tabular}

Table 2: Modified Down and Black scale

\begin{tabular}{|c|c|c|c|c|c|c|c|c|c|c|c|c|c|c|c|c|}
\hline Paper & 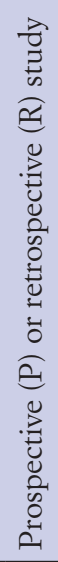 & 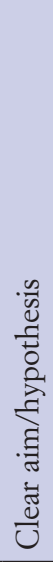 & 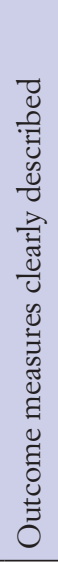 & 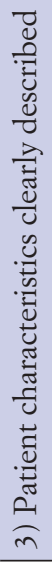 & 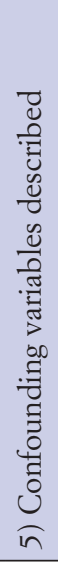 & 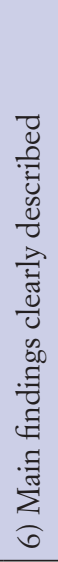 & 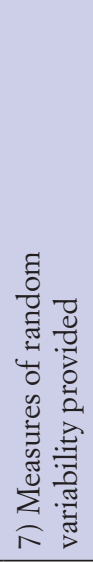 & 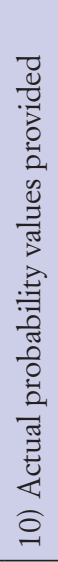 & 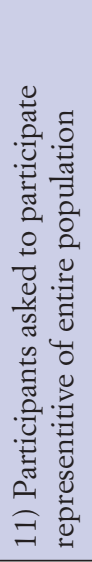 & 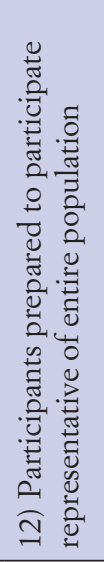 & 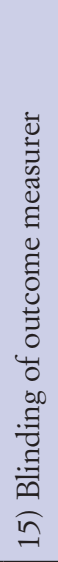 & 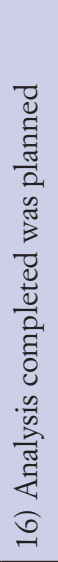 & 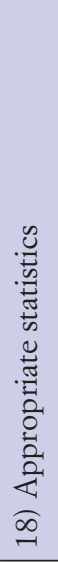 & 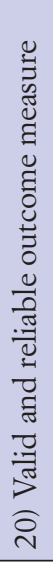 & 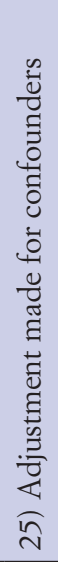 & 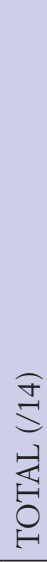 \\
\hline Beers & $\mathrm{R}$ & 1 & 1 & 1 & 1 & 1 & 1 & 1 & 1 & 1 & $\mathrm{U}$ & 1 & 1 & 1 & $\mathrm{U}$ & 12 \\
\hline Fredericson & $\mathrm{R}$ & 1 & 1 & 1 & 1 & 1 & 1 & 1 & 0 & 1 & $\mathrm{U}$ & 1 & 1 & 1 & $\mathrm{U}$ & 12 \\
\hline Ferber R & $\mathrm{P}$ & 1 & 1 & 1 & 1 & 1 & 1 & 1 & 0 & 1 & $\mathrm{U}$ & 1 & 1 & 1 & $\mathrm{U}$ & 11 \\
\hline Foch (2017) & $\mathrm{R}$ & 1 & 1 & 1 & 1 & 1 & 1 & 1 & 0 & 1 & $\mathrm{U}$ & 1 & 1 & 1 & $\mathrm{U}$ & 11 \\
\hline Foch (2014) & $\mathrm{R}$ & 1 & 1 & 1 & 1 & 1 & 1 & 1 & 0 & 1 & $\mathrm{U}$ & 1 & 1 & 1 & $\mathrm{U}$ & 11 \\
\hline Foch (2015) & $\mathrm{R}$ & 1 & 1 & 1 & $\mathrm{U}$ & 1 & 1 & 1 & 0 & 1 & $\mathrm{U}$ & 1 & 1 & 1 & $\mathrm{U}$ & 10 \\
\hline Brown & $\mathrm{R}$ & 1 & 1 & 1 & 1 & 1 & 1 & 1 & 0 & 0 & $\mathrm{U}$ & 1 & 1 & 1 & $\mathrm{U}$ & 10 \\
\hline Grau (2008) & $\mathrm{R}$ & 1 & 1 & 1 & 1 & 1 & 1 & 1 & 0 & 0 & $\mathrm{U}$ & 1 & 1 & 1 & $\mathrm{U}$ & 10 \\
\hline Hamill & $\mathrm{P}$ & 1 & 1 & 1 & 1 & 1 & 1 & 1 & 0 & 1 & $\mathrm{U}$ & 1 & 1 & 0 & $\mathrm{U}$ & 10 \\
\hline Noehren & $\mathrm{P}$ & 1 & 1 & 1 & $\mathrm{U}$ & 1 & 1 & 1 & 0 & 1 & $\mathrm{U}$ & 1 & 1 & 1 & $\mathrm{U}$ & 10 \\
\hline Noehren (2014) & $\mathrm{R}$ & 1 & 1 & 1 & $\mathrm{U}$ & 1 & 1 & 1 & 0 & 1 & $\mathrm{U}$ & 1 & 1 & 1 & $\mathrm{U}$ & 10 \\
\hline Orchard & $\mathrm{R}$ & 1 & 1 & 0 & 1 & 1 & 1 & 1 & 0 & 1 & $\mathrm{U}$ & 1 & 1 & 1 & $\mathrm{U}$ & 10 \\
\hline Grau (2011) & $\mathrm{R}$ & 1 & 1 & 1 & $\mathrm{U}$ & 1 & 1 & 1 & 0 & 0 & $\mathrm{U}$ & 1 & 1 & 1 & $\mathrm{U}$ & 9 \\
\hline Grau (2008) & $\mathrm{R}$ & 1 & 1 & 1 & $\mathrm{U}$ & 1 & 1 & 1 & 0 & 0 & $\mathrm{U}$ & 1 & 1 & 1 & $\mathrm{U}$ & 9 \\
\hline
\end{tabular}


Table 3: Summary of studies included in systematic review

\begin{tabular}{|c|c|c|c|}
\hline Author/year & Study design & Participant details & Protocol \\
\hline Foch $(2017)$ & RCCT & $\begin{array}{l}\mathrm{N}=27 \text { (all female), } 9 \text { ITBFS, } 9 \text { previous } \\
\text { ITBFS, } 9 \text { control } \\
\text { Matched for gender, age }\end{array}$ & 3-D motion analysis during running along a runway \\
\hline Brown (2016) & RCCT & $\begin{array}{l}\mathrm{N}=32 \text { (males and female), } 12 \text { previous } \\
\text { ITBFS, } 20 \text { control. Matched for age. }\end{array}$ & $\begin{array}{l}\text { Kinematicsand kinetic data prior to and following a } \\
\text { run to fatigue }\end{array}$ \\
\hline Foch $(2015)$ & RCCT & $\begin{array}{l}\mathrm{N}=40 \text { (male and female), } 20 \text { previous } \\
\text { ITBFS, } 20 \text { control } \\
\text { Matched for age }\end{array}$ & 3-D motion analysis during running along a runway \\
\hline Foch $(2014)$ & RCCT & $\begin{array}{l}\mathrm{N}=34 \text { (all female), } 17 \text { previous ITBFS, } \\
17 \text { control } \\
\text { Matched for gender and age }\end{array}$ & 3-D motion analysis during running along a runway \\
\hline Noehren (2014) & RCCT & $\begin{array}{l}\mathrm{N}=34 \text { (all male), } 17 \text { ITBFS, } 17 \text { control } \\
\text { Matched for gender, age, mileage, } \\
\text { height, and weight }\end{array}$ & $\begin{array}{l}\text { Hip strength with a hand-held dynamometer, and } \\
3-\mathrm{D} \text { motion analysis data during running at } 3.3 \mathrm{~m} / \mathrm{s} \\
\text { on instrumented treadmill }\end{array}$ \\
\hline Grau S (2011) & RCCT & $\begin{array}{l}\mathrm{N}=36 \text { (males and female), } 18 \text { ITBS, } 18 \\
\text { control } \\
\text { Mean age }-36 \text { (ITBS), } 37 \text { (control) } \\
\text { Matched for gender, height, and weight }\end{array}$ & $\begin{array}{l}\text { Visual 3-D kinematics during barefoot running } \\
(3.3 \mathrm{~m} / \mathrm{s}) \\
7 \text { trial runs, with } 5 \text { runs selected for analysis of } \\
\text { biomechanics } \\
\text { Static kinematic measures taken }\end{array}$ \\
\hline Ferber (2010) & RCCT & $\begin{array}{l}\mathrm{N}=70 \text { (all female), } 35 \text { ITBFS, } 35 \text { control } \\
\text { Matched for age, mileage, and gender }\end{array}$ & $\begin{array}{l}\text { 6-camera } 3 \text {-D motion analysis and force plate. } \\
\text { Subjects ran in neutral running shoes along a } 25 \mathrm{~m} \\
\text { runway at } 3.65 \mathrm{~m} / \mathrm{s}(+/-5 \%) \text { striking a force plate at } \\
\text { the centre }\end{array}$ \\
\hline Hamill J (2008) & PCCT & $\begin{array}{l}\text { N=34 (male and female),17 ITBS, } 17 \\
\text { control } \\
\text { Mean age }-26.8 \text { (ITBS), } 28.5 \text { (control) } \\
\text { Matched for gender, age, and mileage }\end{array}$ & $\begin{array}{l}\text { Visual 3-D kinematic and kinetic data during } \\
\text { running }(3.7 \mathrm{~m} / \mathrm{s}) \\
\text { All subjects used a standard neutral running shoe } \\
5 \text { trial runs were selected }\end{array}$ \\
\hline Grau S (2008) & RCCT & $\begin{array}{l}\mathrm{N}=20 \text { (male and female), } 10 \text { ITBS, } 10 \\
\text { controls } \\
\text { Matched for gender, height, and weight }\end{array}$ & $\begin{array}{l}\text { Concentric, eccentric, and isometric peak torque of } \\
\text { hip abductors and adductors at } 30 \% \mathrm{~s}\end{array}$ \\
\hline Grau S (2008) & RCCT & $\begin{array}{l}\mathrm{N}=73 \text { (male and female), } 21 \text { ITBS, } 52 \\
\text { controls } \\
\text { Matched for gender, height, and weight }\end{array}$ & $\begin{array}{l}\text { Visual 3-D kinematics during barefoot running } \\
(12 \mathrm{~km} / \mathrm{h}) \\
\text { Static kinematic measures taken }\end{array}$ \\
\hline Beers A (2008) & RCCT & $\begin{array}{l}16 \text { (male and female) } \\
\text { All ITBS }\end{array}$ & $\begin{array}{l}\text { 6-week rehab designed to strengthen hip abductors } \\
\text { strength was measured every } 2 \text { weeks using a hand- } \\
\text { held dynamometer and compared bilaterally }\end{array}$ \\
\hline Miller (2008) & RCCT & $\begin{array}{l}\mathrm{N}=16 \text { (gender not stated). } 8 \text { ITBFS, } 8 \\
\text { control } \\
\text { Mean age }-27.5 \text { (ITBFS), } 26.4 \text { (control) } \\
\text { Matched for age }\end{array}$ & $\begin{array}{l}8 \text { camera motion analysis. Participants ran in their } \\
\text { own shoesat selected pace to reach exhaustion in } 20 \\
\text { minutes }\end{array}$ \\
\hline Noehren B (2007) & PCCT & $\begin{array}{l}\text { N=36 (male and female), } 18 \text { ITBS, } 18 \\
\text { controls } \\
\text { Mean age - 26.8 (ITBS), } 28.5 \text { (control) } \\
\text { Matched for gender, age, and mileage }\end{array}$ & $\begin{array}{l}\text { Visual 3-D kinematic and kinetic data during } \\
\text { running }(3.7 \mathrm{~m} / \mathrm{s}) \\
\text { All subjects used a standard neutral running shoe. } 5 \\
\text { trial runs were selected }\end{array}$ \\
\hline Miller (2007) & RCCT & $\begin{array}{l}\mathrm{N}=16 \text { (gender not stated), } 8 \text { ITBFS, } 8 \\
\text { control } \\
\text { Matched for age }\end{array}$ & $\begin{array}{l}8 \text { camera motion analysis. Participants ran in their } \\
\text { own shoesat selected pace to reach exhaustion in } 20 \\
\text { minutes }\end{array}$ \\
\hline Ferber R (2003) & PCCT & $\begin{array}{l}\mathrm{N}=70 \text { (females), } 35 \text { ITBS, } 35 \text { controls } \\
\text { Matched for gender, age, and mileage }\end{array}$ & $\begin{array}{l}\text { Comparisons of hip, knee, and ankle } 3 \text {-dimensional } \\
\text { kinematics and internal moments during the stance } \\
\text { phase of running gait were measured }\end{array}$ \\
\hline
\end{tabular}




\begin{tabular}{|c|c|c|c|}
\hline Author/year & Study design & Participant details & Protocol \\
\hline $\begin{array}{l}\text { Fredericson } M \\
(2000)\end{array}$ & RCCT & $\begin{array}{l}\text { N=54 (male and female), } 24 \text { ITBS, } 30 \\
\text { control } \\
\text { Mean age }-27 \text { (ITBS), } 27.8 \text { (control) } \\
\text { Matched for gender and age }\end{array}$ & $\begin{array}{l}\text { Hip abduction and adduction strengths measured } \\
\text { using a hand-held dynamometer. Five trials on each } \\
\text { leg. Hip abductor strength normalised for weight \& } \\
\text { height. } 6 \text { week standardised rehab }\end{array}$ \\
\hline $\begin{array}{l}\text { Orchard JW } \\
\text { (1996) }\end{array}$ & RCCT & $\begin{array}{l}\mathrm{N}=20 \text { (male and female), } 11 \text { cadavers, } \\
9 \text { ITBS } \\
\text { Mean age }-27(\mathrm{ITBS})\end{array}$ & $\begin{array}{l}\text { Visual 3-D kinematic data during } 2-\mathrm{min} \text { runs on } \\
\text { a treadmill at a constant speed }(2.78-3.89 \mathrm{~m} / \mathrm{s}) \text {. } \\
\text { Subjects used normal footwear. Run repeated with } \\
0.5 \mathrm{~cm} \text { heel-raise on affected side }\end{array}$ \\
\hline Ekman E (1994) & RCCT & $\begin{array}{l}\mathrm{N}=27 \text { (male and female) } \\
10 \text { cadavers, } 7 \text { ITBS, } 10 \text { control }\end{array}$ & $\begin{array}{l}\text { All patients underwent a course of conservative } \\
\text { treatment: MRI of knees in coronal, sagital, and axial } \\
\text { planes. Follow-up MRI in one patient } 1 \text { year on }\end{array}$ \\
\hline
\end{tabular}

Moderate evidence from two high quality studies reported significantly greater knee internal rotation at landing and during running with large effect in individuals who developed ITBFS compared to controls $(E S=1.55,0.91) .(19,20)$ Limited evidence from one high quality study indicates significantly greater femoral external rotation at landing and during running with medium effect in individuals who developed ITBFS compared to controls $(\mathrm{ES}=0.80) .(20)$

Moderate evidence from two high quality studies reported significantly greater rearfoot eversion at landing during running with medium effect in individuals who developed ITBFS compared to controls (ES $=1.18 \& 0.63) .(19,20)$

Moderate evidence from two high quality studies indicates no significant difference in tibial rotation at landing and during running in individuals who developed ITBFS compared to controls. $(19,20)$

\section{Lower-limb kinetics in individuals who develop ITBFS} Limited evidence from one high quality study indicates no significant difference in peak hip abductor moments during running in individuals who developed ITBFS compared to controls $(E S=0.33)$. (20) Limited evidence from one high quality study reported no significant difference in peak knee external rotator moment or peak rearfoot eversion velocity in individuals who developed ITBFS group compared to controls $(\mathrm{ES}=0.32,0.21)$. (20)

\section{Iliotibial band strain in individuals who develop ITBFS}

Limited evidence from one high quality study indicates significantly greater peak ITB strain during running with large effect in individuals who developed ITBFS compared to controls $(\mathrm{ES}=1.88)$. (21) Limited evidence from the same study indicates no significant difference in ITB strain at landing or at maximum knee flexion during running in the injured limb of individuals who developed ITBFS compared to the uninjured limb and compared to controls $(\mathrm{ES}=0.44 \& 0.37) .(21)$

\section{Case-control studies}

\section{Lower-limb kinematics in individuals with ITBFS}

Strong evidence from three high quality studies indicates significantly less peak hip adduction during running with medium effect in ITBFS participants compared to controls $(\mathrm{ES}=1.03 \& 0.98) .(10,17,18)$ Limited evidence from one high quality study indicates no significant difference in hip adduction during running in ITBFS participants compared to controls $(\mathrm{ES}=0.29)$. (11)

Limited evidence from two high quality studies separately reported significantly greater tibial internal rotation and significantly less tibial internal rotation at landing and through stance during running in ITBFS participants compared with controls, however inadequate data were available for effect size calculations. $(11,17)$

Moderate evidence from two high quality study indicates significantly reduced rearfoot eversion during running in ITBFS participants compared to controls with small effect $(\mathrm{ES}=1.18$ \& 1.14).(17,27) Limited evidence from one high quality study indicates no significant difference in rearfoot motion during running in ITBFS participants compared to controls ( $\mathrm{ES}=0.33)$.(18) Limited evidence from one high quality study indicates no significant differences in hip, knee, and ankle flexion and extension during running in ITBFS participants compared to controls $(\mathrm{ES}=0.24$, 0.38 , \& 0.00).(18) Moderate evidence from two high quality studies also reported no significant differences in hip, knee, and ankle flexion and extension during running in ITBFS participants compared to controls, however inadequate data was available for effect size calculations. $(17,26)$

\section{Lower-limb kinetics in individuals with ITBFS}

Strong evidence from four high quality studies indicates significantly lower hip abductor moments during isometric exercises with large effect in ITBFS participants compared to controls $(\mathrm{ES}=1.20,1.40,2.25)$. $(10,17,18,24)$. Moderate evidence from two high quality studies indicates no signifi- 
cant difference in hip abductor moments during isometric exercises in ITBFS participants compared to controls (ES = $0.33 \& 0.3) .(11,20)$

Moderate evidence from two high quality studies indicates significantly lower hip abductor moments during isometric exercises with medium effect on the injured compared to uninjured side in ITBFS participants $(\mathrm{ES}=1.43,0.77,1.02) .(23,24)$ Limited evidence from one study indicates significantly lower hip, knee, and ankle flexion moments in the ITBFS group compared with controls (ES=1.02, 0.69, \& 0.67). (18) Limited evidence from one high quality study reported no significant differences in peak rearfoot peak motion moments between ITBFS participants and controls $(\mathrm{ES}=0.04) .(18)$

\section{Conservative intervention Longer-term effects}

Limited evidence from one study reported ITBFS resolution and no recurrence at six months following a 6-week rehabilitation programme.(24) Moderate evidence from two high quality studies indicates significantly greater hip abductor moments in injured compared to uninjured limbs in individuals with ITBFS following a 6-week rehabilitation programme $(\mathrm{ES}=-2.88,-0.63) .(23,24)$ Limited evidence from one high quality study indicates a significant decrease in pain in individuals with ITBFS group following a 6-week rehabilitation programme (ES=-1.57).(23) Ekman investigated the effects of combined physical therapy, NSAIDs, and stretching exercises. (25) The study found that 100\% of ITBFS participants reported less pain following a course of conservative treatment, and $86 \%$ had complete resolution of symptoms at 8-weeks.(25) Limited evidence from one high quality study reported no immediate change in pain or knee flexion angles with a heel rise in injured limbs of individuals with ITBFS (ES=0.10).(26)

\section{DISCUSSION}

This systematic review provides a clinically relevant summary on lower limb biomechanical risk factors and changes associated with ITBFS, and the efficacy of conservative treatment. Our study extends work by previous systematic reviews (5-7) by drawing on important recent trials concerning biomechanical analysis, by combining literature on the efficacy of interventions targeting lower-limb biomechanics, and by pooling data for effect size calculations. A total of 18 studies met the inclusion criteria and were evaluated.

Moderate evidence from this meta-analysis indicates greater knee internal rotation during running represents a proximal risk factor for ITBFS. Limited evidence indicates greater hip adduction and greater femoral external rotation repre- sent proximal risk factors for ITBFS. Moderate evidence from this meta-analysis indicates greater rearfoot eversion at landing during running represents a distal risk factor for ITBFS. Considering this, greater hip adduction and greater rearfoot eversion appear to be risk factors which are reduced by ITBFS patients, possibly to lower pain associated with ITB strain and ITB friction.

Moderate evidence from this meta-analysis indicates a six-week rehabilitation programme involving NSAID prescription, ITB stretching, and hip abductor strengthening reduces pain and prevents recurrence for up to six months. Improvements in pain and outcome may be explained by changes in kinematic and kinetic risk factors. Eleven studies in this review presented adequate data to complete effect size calculations. $(10,11,17-21,23,24,26,27)$ Five new studies were identified following Ellis's review investigating lowerlimb biomechanics and treatment for ITBFS.(11) No previous meta-analysis has evaluated the effects of lower-limb biomechanics and efficacy of conservative treatment in individuals with ITBFS.

\section{Theory behind ITBFS}

The ITB is a sheet of connective tissue lying level or just anterior to the LFE at extension. (4) Studies have reported antero-posterior translation $(4,25,28)$ and medio-laterally shift (4) of the ITB fibres at the LFE during knee flexion. One common hypothesis suggests the presence of a bursa between the LFE and ITB in cadaveric controls.(25) In pathological ITBFS, MR imaging and cadaveric dissection shows the bursa may become inflamed and fluid-filled as a result of chronic friction against the ITB.(25) However, a second hypothesis suggests an absence of a true bursa between the LFE and ITB, and histological inspection reports a layer of highly vascularised and innervated adipose tissue with localised inflammation. $(4,28)$ The evidence suggests ITBFS may be a result of repeated compression against the adipose tissue or fat pad, analogous to Hoffa's syndrome in the knee. Consequently, compression against the fat pad from repetitive movement (e.g. running or pedaling) may produce pain over the LFE associated with ITBFS. While these represent hypotheses behind ITBFS, a number of proximal, distal, and local mechanisms are involved.

\section{Possible Mechanisms \\ Local}

Evidence from case-control studies found that ITBFS participants exhibited a thicker ITB over the LFE compared with controls. $(25,26)$ This is likely to be an effect of chronic friction against the LFE rather than a cause of ITBFS. 
A

\begin{tabular}{|c|c|c|c|c|c|c|c|}
\hline \multirow[b]{2}{*}{ Study or Subgroup } & \multicolumn{3}{|c|}{ ITFBS } & \multicolumn{2}{|c|}{ Control } & \multicolumn{2}{|r|}{ Std. Mean Difference } \\
\hline & Mean & SD & Total & Mean & SD & Total & IV, Fixed, $95 \% \mathrm{CI}$ \\
\hline \multicolumn{8}{|c|}{ 2.2.1 Hip Abduction Moment } \\
\hline Noehren 2007 & -1.4 & 0.37 & 18 & -1.3 & 0.19 & 18 & $-0.33[-0.99,0.33]$ \\
\hline \multicolumn{8}{|c|}{ 2.2.2 Knee External Rotation Moment } \\
\hline Noehren 2007 & -0.12 & 0.12 & 18 & -0.09 & 0.05 & 18 & $-0.32[-0.98,0.34]$ \\
\hline \multicolumn{8}{|c|}{ 2.2.3 Rearfoot Inversion Moment } \\
\hline Noehren 2007 & -0.15 & 0.1 & 18 & -0.13 & 0.09 & 18 & $-0.21[-0.86,0.45]$ \\
\hline
\end{tabular}

B

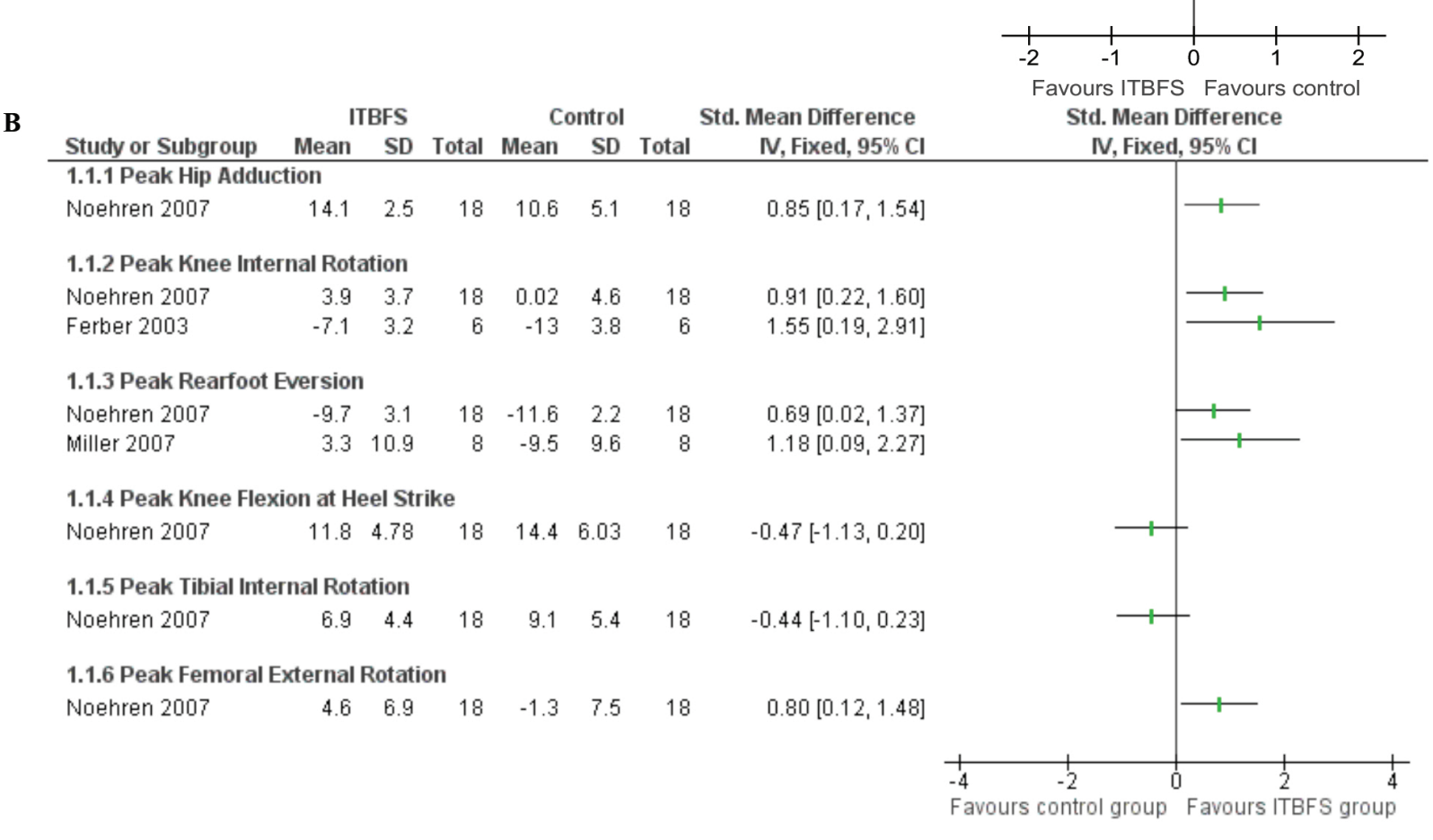

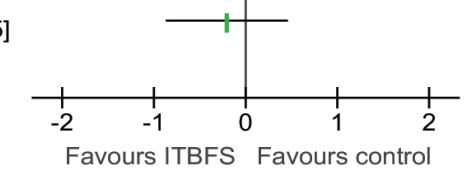

IV, Fixed, $95 \% \mathrm{CI}$

C

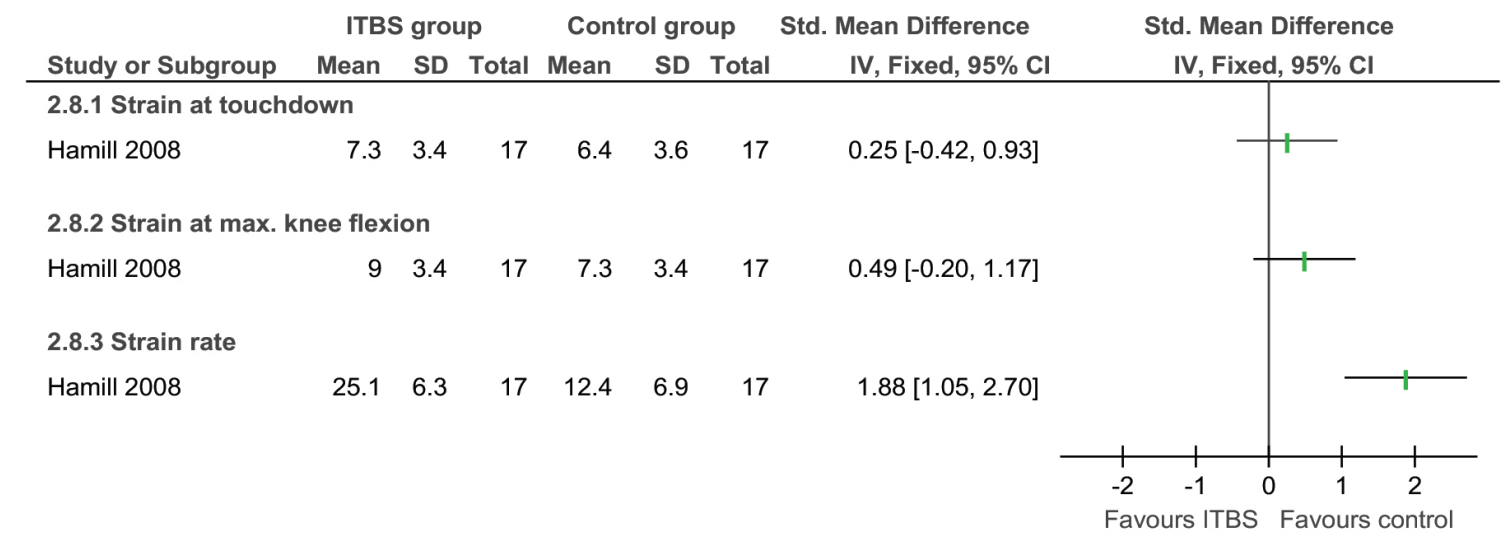

Figure 2. Prospective results: lower-limb kinematics (A); kinetics (B); and ITB strain rate between ITBFS and control groups (C). 
A

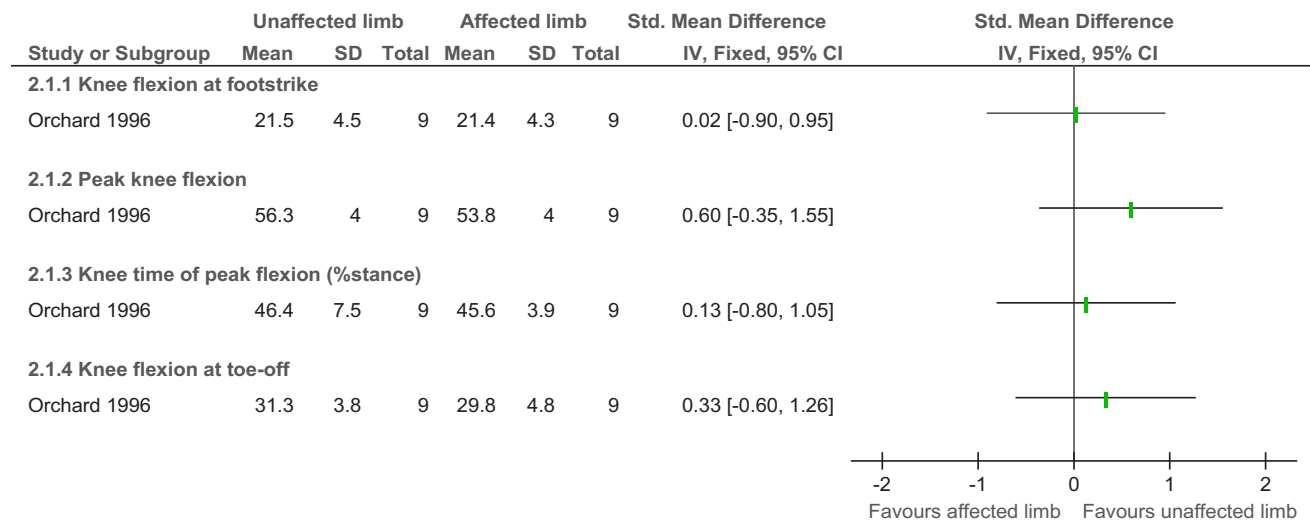

B

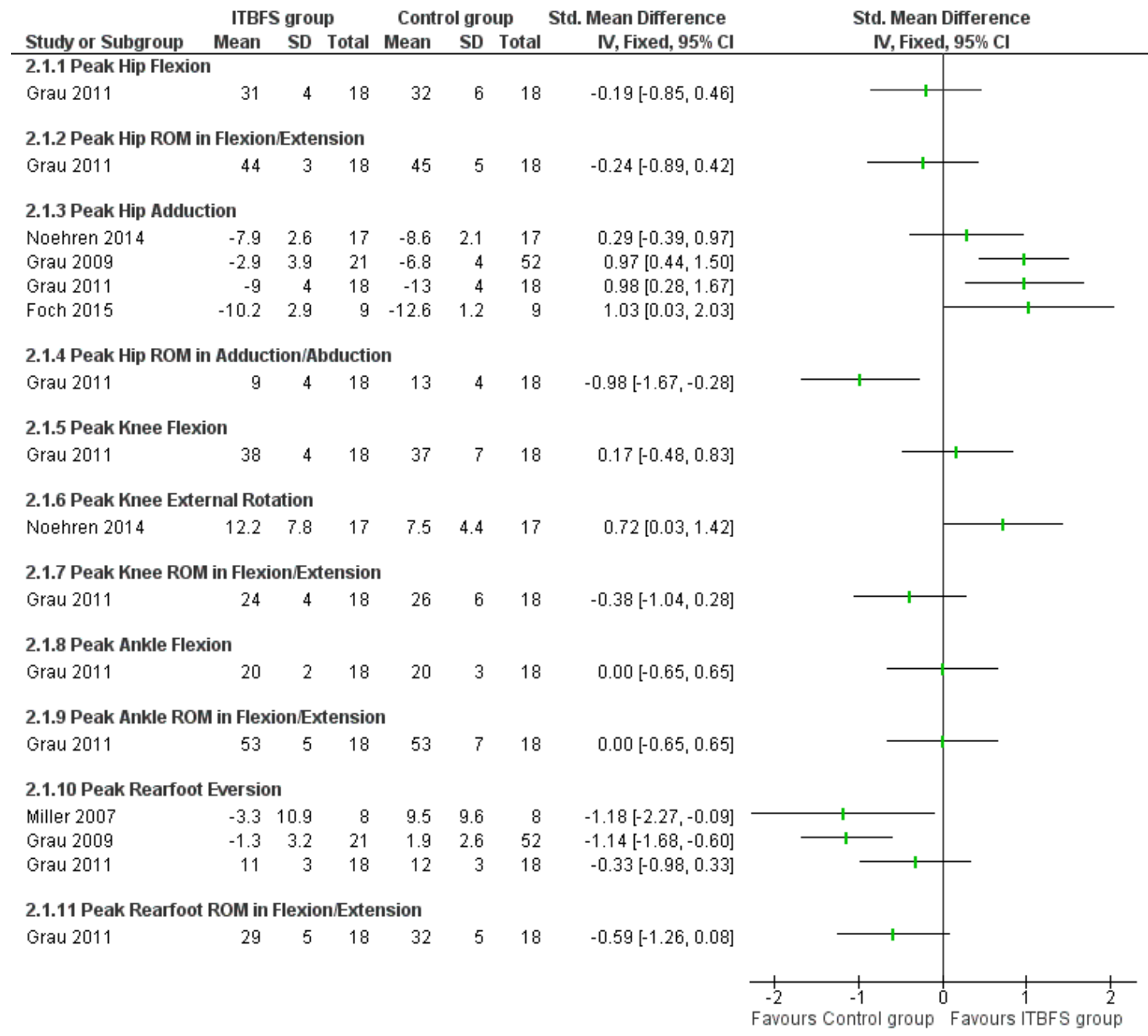

Figure 3. Case-control results: lower-limb kinematics between injured and uninjured limbs in ITBFS participants (A); and between ITBFS and control groups (B). 


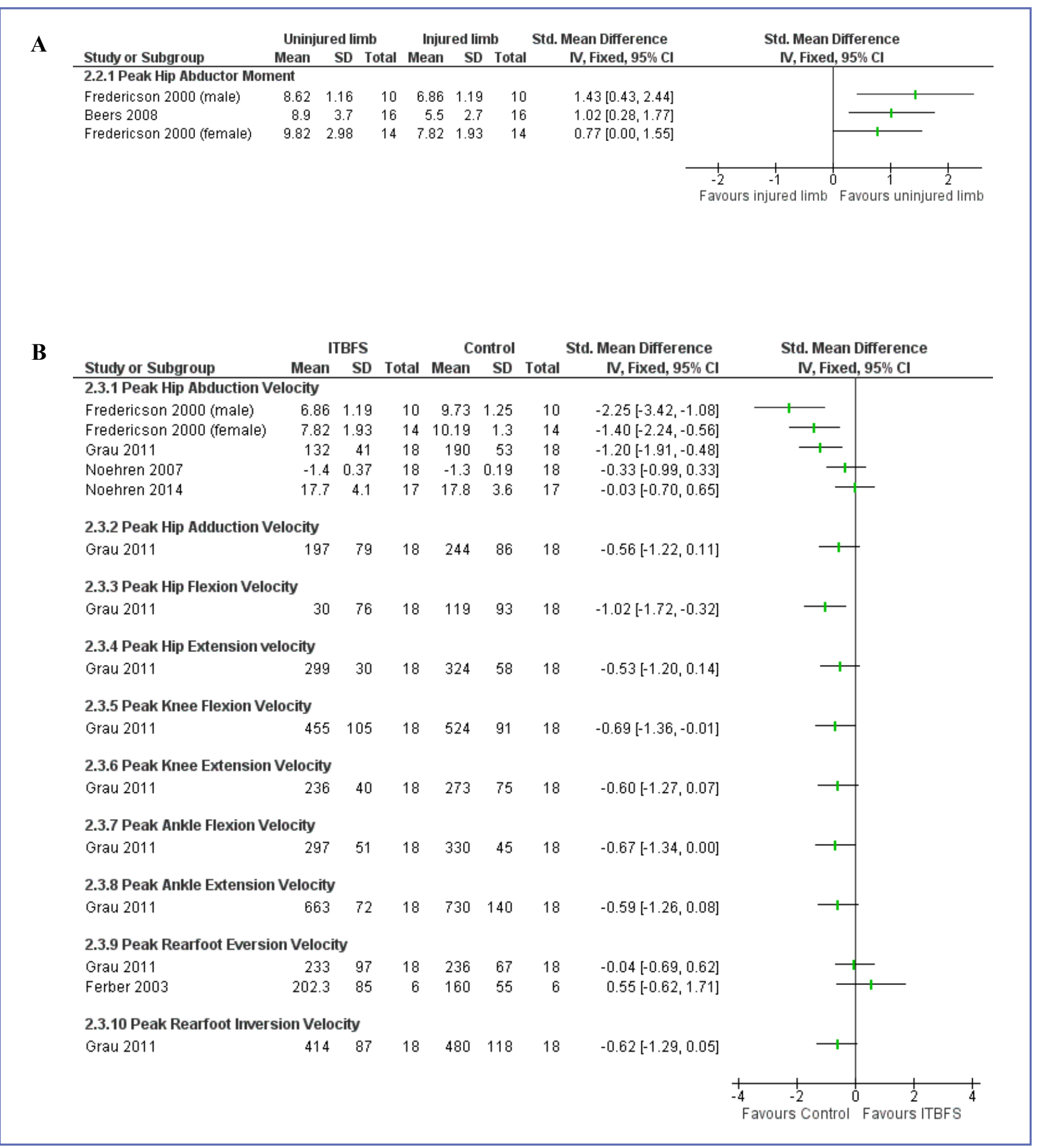

Figure 4. Case-control results: lower-limb kinetics between injured and uninjured limbs in ITBFS participants (A); and between ITBFS and control groups (B). 


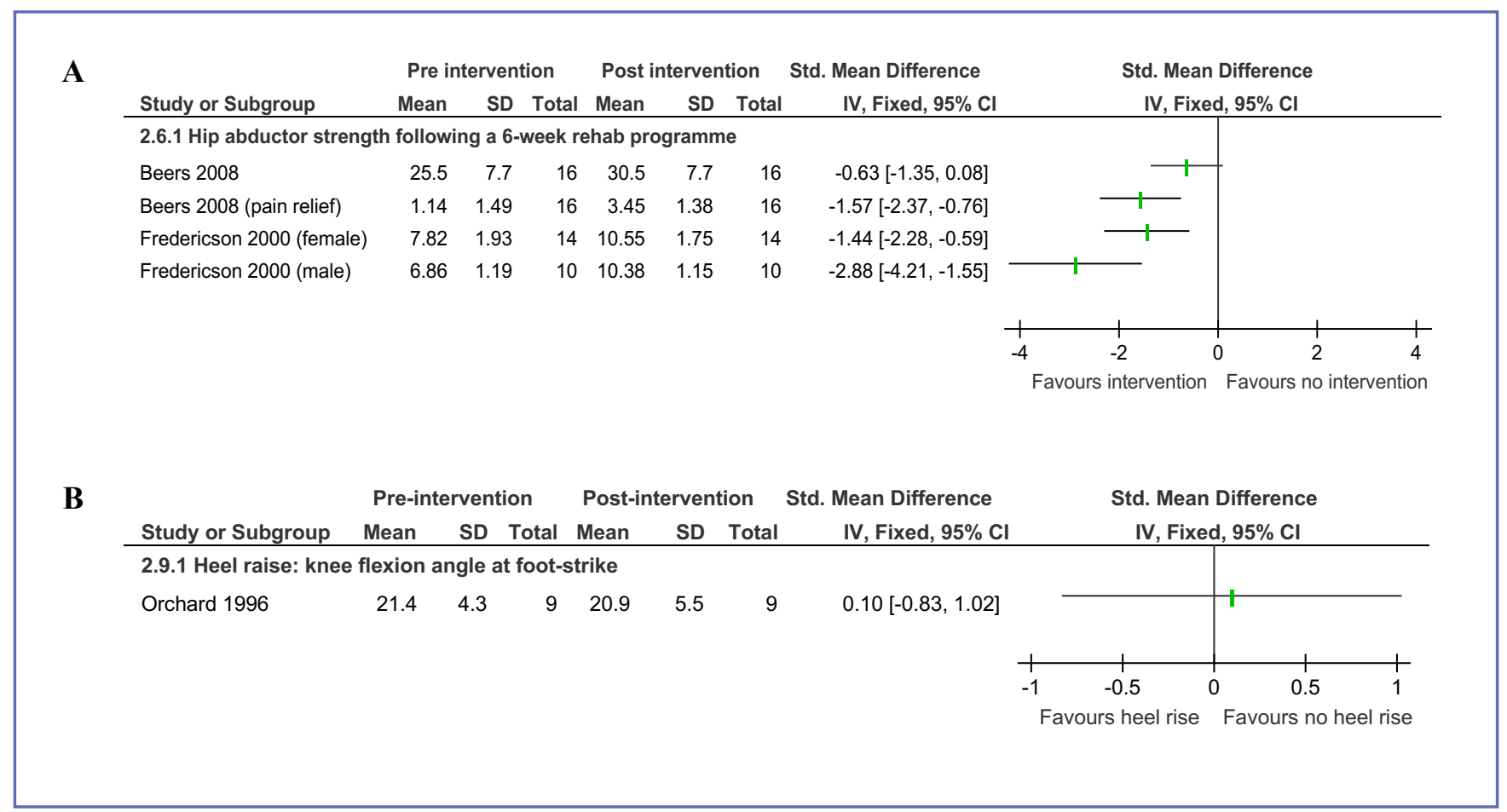

Figure 5. Conservative treatment in ITBFS participants. Mid-term changes in hip abductor strength (A); and immediate changes in knee flexion angle following a heel raise (B).

Chronic antero-posterior friction may cause changes in the connective tissue, formation of a bursa, and an increase in ITB thickness resulting in ITBFS. While this theory is widely regarded as the local mechanism of ITBFS, further investigation is required in-light of recent conflicting evidence.

Evidence from histological studies suggests the presence of Pacinian corpuscles in the layer of adipose tissue beneath the ITB. (4) Repeated antero-posterior translation on the LFE combined with medio-lateral compression of the fat pad may result in localised inflammation and pain associated with ITBFS. Pacinian corpuscles represent mechanoreceptors responsible for detecting gross changes in vibration and pressure, and hypertrophy as a result of localised inflammation has been shown to cause pain. (4) Further investigation of this hypothesis alone, and in combination with chronic antero-posterior friction is required to identify how local factors may contribute to the development of ITBFS.

\section{Proximal}

Greater hip adduction (20) is a proximal risk factor for ITBFS, which is reduced by patients, $(17,18)$ resulting in a loss of hip abductor strength when compared to the uninjured side and controls. $(18,23,24)$ One prospective study concluded that strain rate may be a causative factor as no significant differences in magnitude of strain between ITBFS and control participants were found, (20) One common hypothesis suggests that increased hip adduction may move the attachment of the ITB medially, increasing ITB strain and its compression against the LFE and fat pad. $(2,28)$ It appears that ITBFS participants with greater hip adduction compensate to help reduce ITB strain rate and associated pain. While this hypothesis represents a possible proximal mechanism of ITBFS, further investigation into ITB strain and strain rate is required considering recent evidence.

Greater knee internal rotation and greater femoral external rotation at footstrike and through stance represents a proximal risk factor for ITBFS $(19,20)$ which is reduced by patients with ITBFS. (18) One hypothesis suggests that greater femoral ER may be a result of weakness of the muscles at the hip (gluteus minimus, anterior fibers of gluteus medius, and tensor fascia latae), leading to an increase in LFE friction and fat pad compression by the ITB. However, prospective studies have reported that hip abductor torque is not a risk factor for ITBFS. It is possible that differences in muscle onset timing rather than magnitude may contribute, and there is currently no published literature investigating electromyographic $(\mathrm{EMG})$ onset timing in ITBFS. This needs addressing considering muscle activity may represent a possible proximal mechanism in ITBFS. 


\section{Distal}

Greater rearfoot eversion at foot strike represents a distal risk factor $(20,27)$ which is reduced by patients with ITBFS. $(18,19)$ In one study a subgroup of ITBFS participants who demonstrated highest rearfoot eversion also demonstrated high tibial IR.(19) Rearfoot eversion is coupled with tibial IR,(30) and one hypothesis suggests that an increase in these variables may result in increased ITB strain and fat pad compression. $(2,4)$ ITBFS participants with greater rearfoot eversion appear to compensate to help reduce their ever-sion, tibial IR, ITB strain, and fat pad compression. Consid-ering these implications, further investigation into the effi-cacy of conservative interventions that can alter and address rearfoot biomechanics is needed.

\section{Conservative therapy considerations}

A six-week rehabilitation programme involving NSAID prescription, ITB stretching, and hip abductor strengthening appears to effectively reduce pain and prevent recurrence for up to six months. (24-26) Evidence suggests that mechanisms of efficacy may be: i) reduced fat pad inflammation (NSAIDs), ii) reduced ITB strain or strain rate (ITB stretching), and iii) reduced fat pad compression (hip abductor strengthening). This review highlights the presence of local, proximal, and distal mechanisms behind ITBFS. To achieve the greatest benefits, interventions targeting patient-specific lower-limb biomechanics are required. Further investigation of the efficacy of conservative interventions that target lower-limb biomechanics such as taping, orthoses, and gait retraining are also required.

\section{Quality assessment}

Methodological quality of biomechanical studies was assessed using a modified Down and Black scale, and scores ranged between nine and twelve out of 14. Many studies failed to suitably describe participant characteristics, if participants were representative of the entire population, and details of participant matching. Matching of running participants has been shown to be better at identifying between group differences in biomechanical variables. (17) Intervention studies were assessed using the PEDRO scale, and scores ranged between five and seven out of 11 . Therapist blinding is difficult to achieve in physical therapy studies as treatments require therapist skill, knowledge, and instruction, however, none of the studies chose to blind subjects or assessors which creates potential for bias.

A variety of study designs were used: three prospective and eleven case-control. Each design presents specific advantages but comes with certain disadvantages. Prospective studies establish cause and effect relationships, however, these studies are more costly with regards to participant recruitment and follow-up. $(19,20)$ The review highlights the inconsistencies between prospective and retrospective data, suggesting that ITBFS participants may change lower-limb biomechanics as a result of injury. The evidence suggests that case-control studies are not adequate for all variables, therefore future studies should focus on prospective design.

\section{Limitations and future research}

Three prospective studies investigated lower-limb biomechanics, however, different kinetic variables were assessed. While causative factors were reported, further prospective research investigating the same variables is required to confirm findings from these studies. There is currently a paucity of research evaluating structural factors such as a prominent LFE, leg-length discrepancies, or varus alignment as mechanisms behind ITBFS. Prospective studies investigating these variables are needed to better understand the influence of local structural factors on ITBFS. Evidence from this review suggests that differences in hip abductor timing rather than magnitude may contribute to ITBFS. There is currently no published literature investigating electromyographic (EMG) onset timing in ITBFS participants. Prospective EMG studies are needed to understand the influence of neuromuscular function on ITBFS. There are currently no clinical prediction rule (CPR) studies investigating which patients respond successfully to conservative treatments such as hip abductor strengthening or ITB stretches. ITBFS is a condition of heterogeneous nature, and clinical prediction studies based on ITBFS can help to identify subgroups of patients who respond to specific treatments. This review supports the need for CPR based on functional aspects of ITBFS (perceived pain, neuromuscular control, and lower-limb biomechanics) to be developed to aid practitioners. A six-week rehabilitation programme involving NSAID prescription, ITB stretching, and hip abductor strengthening appears to effectively reduce pain and prevent recurrence for up to six months. Further long term ( $>12$ months) RCTs evaluating this programme, and other conservative interventions targeting biomechanical factors associated with ITBFS, including taping, foot orthoses and gait retraining are required. Furthermore, the effects of specific intervention programmes aimed at targeting patients with specific proximal, local, or distal mechanisms of ITBFS should be evaluated to aid practitioners.

\section{CONCLUSION}

Greater hip adduction, greater knee internal rotation, and greater femoral external rotation represent possible proximal risk factors for ITBFS. Greater rearfoot eversion represents a distal risk factor for ITBFS. Considering this, greater hip adduction, knee internal rotation, and rearfoot eversion are risk factors which are reduced by ITBFS patients to lower ITB 
strain and associated pain. Addressing the absence of research evaluating lower limb electromyography is needed to understand the influence of muscle function on ITBFS. A six-week rehabilitation programme involving NSAID prescription, ITB stretching, and hip abductor strengthening appears to effectively reduce pain and prevent recurrence for up to six months. Further long term (>12 months) RCTs evaluating

\section{REFERENCES}

1. Noble C. Iliotibial band friction syndrome in runners. Am J Sports Med. 1980;8:232-234.

2. Farrell KC, Reisinger KD, Tillman MD, et al. Force and repetition in cycling: possible implications for iliotibial band friction syndrome. The Knee. 2003;10:103-109.

3. Orava S. Iliotibial tract friction syndrome in athletes-an uncommon exertion syndrome on the lateral side of the knee. Br J Sports Med 1978;12(2):69-73.

4. Fairclough J, Hayashi K, Toumi H, et al. The functional anatomy of the iliotibial band during flexion and extension of the knee: implications for understanding iliotibial band syndrome. J Anat. 2006;208:309-316.

5. Louw M, Deary C. The biomechanical variables involved in the aetiology of iliotibial band syndrome in distance runners - A systematic review of literature. Phys Ther Sport. 2014;15(1);64-75.

6. Ellis R, Hing W, Reid R. Iliotibial band friction syndrome-A systematic review. Manual Th. 2007;12:200-208.

7. Worp MP, Horst N, A Wijer, et al. Iliotibial band syndrom ine runners. A systematic review.

8. Foch E, Westbrooks JW, Milner CE. Hip Neuromechanics In Women With And Without Previous Iliotibial Band Syndrome During A 30-minute Run. Med \& Sci SEM. 2017:49(5S);995.

9. Brown AM, Zifchock RA, Hillstrom HJ, et al. The effects of fatigue on lower extremity kinematics, kinetics and joint coupling in symptomatic female runners with iliotibial band syndrome. Clin Biomech (Bristol, Avon). 2016 Nov;39:84-90

10. Foch E, Reinbolt JA, Zhang S, et al. Associations between iliotibial band injury status and running biomechanics in women. Gait Posture. 2015 Feb;41(2):706

11. Noehren B, Schmitz A, Hempel R, et al. Assessment of Strength, Flexibility, and Running Mechanics in Males with Iliotibial Band Syndrome. J Orthop Sports Phys Ther. 2014 Mar; 44(3): 217-222

12. Foch E, Milner CE. The influence of iliotibial band syndrome history on running biomechanics examined via principal components analysis. 2014:47(1);81-86.

13. Moher D, Liberati A, Tetzlaff J, Altman DG, The PRISMA Group (2009). Preferred Reporting Items for Systematic Reviews and Meta-Analyses: The PRISMA Statement. BMJ 2009;339

14. Maher CG, Sherrington C, Herbert RD. Reliability of the PEDro Scale for Rating Quality of Randomized Controlled Trials. Phy Th. 2003;83(8):713-721.

15. Barton CJ, Munteanu SE, Menz HB, et al. The Efficacy of Foot Orthoses in the Treatment of Individuals with Patellofemoral Pain Syndrome. A Systematic Review. Sports Med 2010; 40 (5): 377-395 this programme and other conservative interventions targeting biomechanical factors associated with ITBFS, including taping, foot orthoses and gait retraining are required.

\section{Conflict of Interest}

The authors declare that they have no conflict of interest

16. van Tulder M, Furlan A, Bombardier C, Bouter L. Updated method guidelines for systematic reviews in the Cochrane Collaboration Back Review Group. Spine (Phila Pa 1976) 2003;28:1290-1299

17. Grau S, Maiwald C, Krauß I, et al. The influence of matching populations on kinematic and kinetic data in runners with ilio-tibial band syndrome (ITBS).

18. Grau S, Krauss I, Maiwald C, et al. Kinematic classification of iliotibial band syndrome in runners. Scand J Med Sci Sports. 2011; 21:184-189.

19. Ferber RI, Davis M, Hamill J, et al. Prospective Biomechanical Investigation of Iliotibial Band Syndrome in Competitive Female Runners. Med Sci in Sports Exerc. 2003;35(5):91.

20. Noehren B, Davis I, Hamill, J. ASB clinical biomechanics award winner 2006 prospective study of the biomechanical factors associated with iliotibial band syndrome. Clin Biom. 2007;22(9):951-6.

21. Hamill J, Miller R, Noehren B, et al. A prospective study of iliotibial band strain in runners. 2008;23:1018-1025.

22. Grau S, Krauss I, Maiwald C, et al. Hip Abductor Weakness is not the Cause for Iliotibial Band Syndrome. Int J Sports Med. 2008;29(7):579-583.

23. Beers A, Ryan M, Kasubuchi Z, et al. Effects of Multi-modal Physiotherapy, Including Hip Abductor Strengthening, in Patients with Iliotibial Band Friction Syndrome. Physio Canada. 2008;60(2):180-188.

24. Fredericson MD, Cookingham CL, Chaudhari AM, et al. Hip Abductor Weakness in Distance Runners with Iliotibial Band Syndrome. Cl J of Sport Med. 2000;10(3):169-175.

25. Ekman EF, Pope T, Martin DF. Magnetic Resonance Imaging of Iliotibial Band Syndrome. Am J Sports Med. 1994;22(6): 851-854

26. Orchard JW, Fricker PA, Abud AT, et al. Biomechanics of iliotibial band friction syndrome in runners. Am J Sports Med. 1996;24(3):375-9

27. Miller RH, Lowry JL, Meardon SA, et al. Lower extremity mechanics of iliotibial band syndrome during an exhaustive run. Gait Posture. 2007;26(3):407-13

28. Muhle C, Ahn JM, Yeh L, et al. Iliotibial band friction syndrome: MR imaging findings in 16 patients and MR arthorographic study of six cadaveric knees. Radiology. 1999;212:103-110.

29. Orava, S., 1978. Iliotibial tract friction syndrome in athletes - an uncommon exertion syndrome on the lateral side of the knee. British Journal of Sports Medicine 12, 69-73.

30. Lundberg A, Svensson OK, Bylund C, et al. Kinematics of the ankle/foot complex - Part 2: Pronation and supination. Foot Ankle. 1989;9:248-253. 